\title{
Should HPV vaccination be gender neutral?
}

$\mathrm{H}$ uman papillomavirus (HPV) doesn't discriminate based on gender and neither should policy-makers, say health experts. Nova Scotia recently followed the lead of Prince Edward Island and Alberta in introducing HPV vaccination for boys, but the majority of Canadian provinces continue to immunize only girls, primarily because of the cost.

Jeroen Luyten, a health policy expert at the London School of Economics and Political Science, and other advocates for gender-neutral HPV immunization say the strongest case can be built on ethical and not financial grounds.

Gender is a blunt tool for deciding who gets the vaccine, says Luyten. Men are at lower risk, on average, of acquiring the virus but some subgroups, including men who have sex with men, may be at higher risk than girls.

"Compare this to only targeting AIDS prevention at black people because, on average, they have a higher risk of getting infected with HIV," says Luyten.

According to the World Health Organization, HPV infection is the world's most common sexually transmitted infection. It causes most cases of cervical cancer, a disease that resulted in an estimated 266000 deaths worldwide in 2012. HPV infection is also responsible for genital warts, and some oropharyngeal, anal and penile cancers. By the end of 2013, there were 55 countries with HPV vaccination programs.

In Canada, girls are offered free immunization. Only three provinces offer it to boys, although Ontario is reviewing its stance.

The Ontario Ministry of Health and Long-Term Care told CMAJ that, after its review of the HPV program, it will determine whether additional changes are required. Factors being considered include burden of disease, vaccine effectiveness, cost-effectiveness, and the impact on the health system.

The National Advisory Committee on Immunization recently changed its position. In February 2015, it expanded

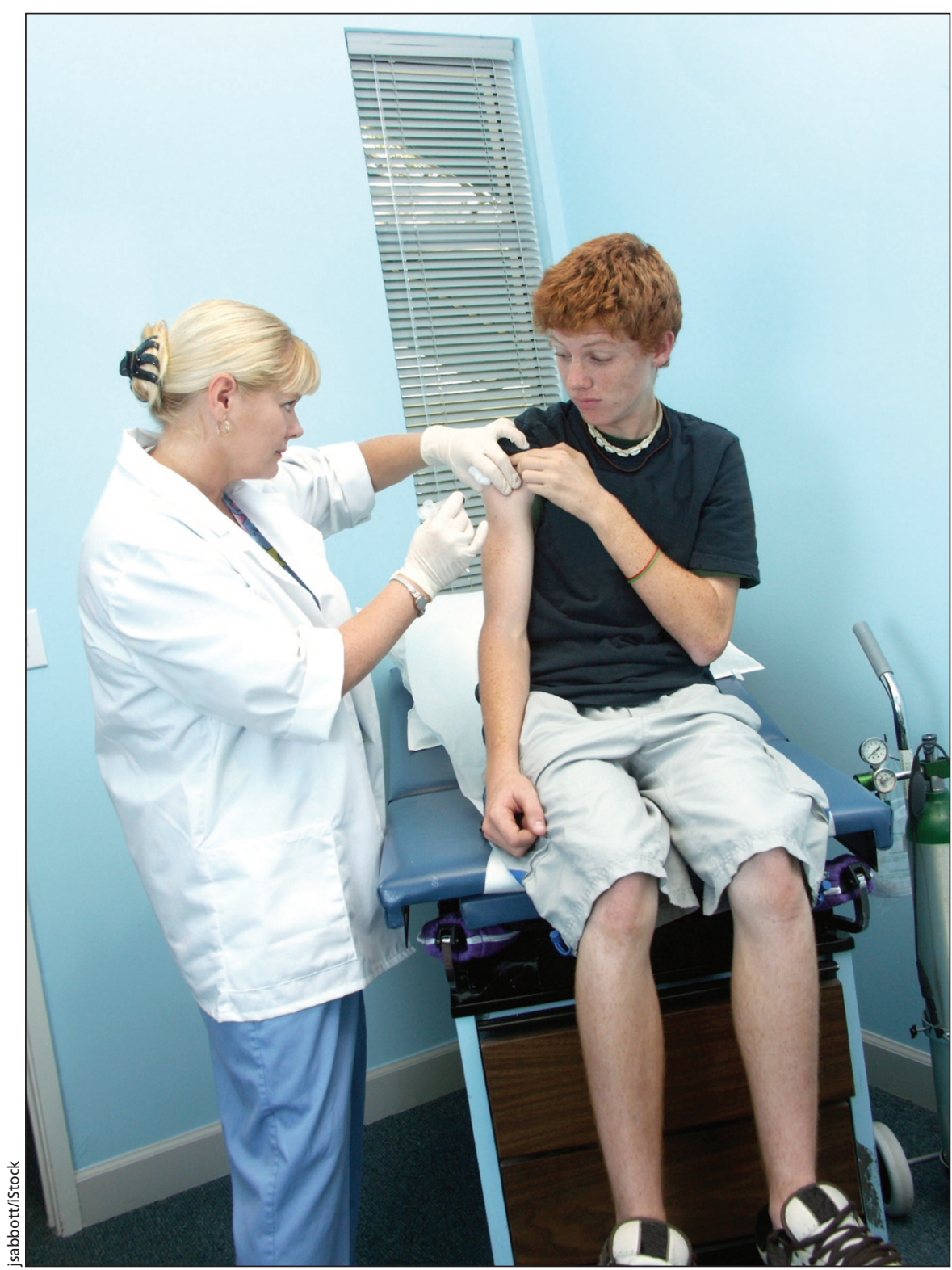

Experts say the decision to vaccinate boys should be based on fairness, not finances.

its recommendation to include HPV immunization for males aged 9-26. Although it used to recommend the three-dose treatments, the committee now says a two-dose schedule may be considered for those under 15 to allow for potential cost savings. Currently, the cost per dose for recombinant human papillomavirus vaccine types $6,11,16$ and 18 (Gardisil) is about $\$ 140$, or $\$ 420$ for a three-dose course.
The committee states that a two-dose regimen may also increase the vaccine's acceptability to consumers and lead to improved uptake.

The Federation of Medical Women in Canada has been calling for boys to be vaccinated for HPV since 2011 . Dr. Vivien Brown, the association's incoming president, says policy-makers in all provinces appear to favour genderneutral immunization and aren't neces- 
sarily driven by partisan politics. But she added that, ultimately, it all comes down to money.

"What I soon found was that a lot of the decisions end up getting made by the treasurer," said Brown.

Evidence is beginning to emerge, however, that the upfront cost of immunization may later be offset by disease prevention. A recent study in Cancer showed that it may be cost-effective to vaccinate school-age boys against HPV as a strategy for preventing cancers later in life.

Both Brown and Luyten say that, regardless of the costs, the decision to vaccinate boys should be primarily about saving lives. They are also challenging policy-makers to consider how to best protect people from disadvantaged social groups who will remain unvaccinated because of gaps in immunization programs.

Whenever there is a life-saving measure, it has to be equitable in terms of its distribution, says Brown. "We now have excellent evidence that this decreases the risk of various cancers, whether we're talking about women or men, and so there has to be equal access. That way,
Canadians can make their own decision for themselves and their families about whether they want to be immunized."

Vaccination is not just a right, it can also be a social duty and obligation, adds Luyten. "If we consider it extrablameworthy to infect sexual partners, as compared to complete strangers, then there is an additional reason to subsidize the vaccine, as it would enable individuals to act morally and perform according to their duty."- Martin Smith, CMAJ

CMAJ 2015. DOI:10.1503/cmaj.109-5066 